\title{
Evaluation of oral hygiene and perception of patients undergoing orthodontic treatment attending OPD at Terna Dental College, Mumbai, Maharashtra
}

\author{
Krina Shah ${ }^{1}$, Shailesh Shenava ${ }^{2}$, Rohit Kulshrestha ${ }^{3, *}$, Chinmaya Hawaldar ${ }^{4}$ \\ ${ }^{1}$ Dental Surgeon, ${ }^{2}$ Professor and Head, ${ }^{3}$ Senior Lecturer, ${ }^{4}$ PG Student, ${ }^{1}$ Dept. of Public Health Dentistry, ${ }^{2-4}$ Dept. of Orthodontics and \\ Dentofacial Orthopedics, Terna Dental College and Hospital, Navi Mumbai, Maharashtra, India
}

*Corresponding Author: Rohit Kulshrestha

Email: kulrohit@gmail.com

\begin{abstract}
Aim: To evaluate oral health awareness and hygiene practices among patients undergoing fixed orthodontic treatment.

Materials and Methods: A total of 500 patients in the age group of 15-35 years who had permanent dentition were selected using random sampling technique. A self-administered structured questionnaire including 15 multiple choice questions was given to them. The results were analysed using percentage.

Results: The results of the study showed that nearly $62 \%$ patients did not follow the oral hygiene instructions, and despite being given instructions, most of them hesitated in practicing them consistently. An excellent finding was that all the patients used the toothbrush as an aid used to clean teeth.

Conclusion: The knowledge and attitude of patients towards oral hygiene among orthodontic patients was minimal. Non-maintenance of oral health practices can be due to many factors. To name a few, may be ignorance and lack of motivation. Hence, intense oral hygiene programs should be established and should be inspected in the future.
\end{abstract}

Keywords: Fixed orthodontic treatment, Oral hygiene.

\section{Introduction}

Oral Health is the window to systemic disease. Sometimes, Systemic diseases remain undiagnosed or untreated because of this missing oral hygiene awareness. ${ }^{1,2}$ Oral hygiene is important to maintain healthy dentition and oral cavity and prevent caries, halitosis, xerostomia, cold sores and tempro-mandibular disorder. ${ }^{3}$ Orthodontic treatment has great impact on dentofacial complex. Thus, it is widely practiced branch of dentistry. ${ }^{4}$ Majority people don't regular brush teeth after principal meals. Thus, there is food lodgement. ${ }^{5}$ According to previous studies there is a rapid fall in oral hygiene compliance after the initial bonding, and the appliance favours plaque build-up and it is a hindrance to the hygiene practice like tooth brushing and flossing. ${ }^{6}$ This can even lead to gingivitis. Enamel decalcification may occur due to plaque build-up around the bracket base. Orthodontic patients are advised to rinse two times a day with $20 \mathrm{~mL}$ of Listerine as an adjunct to brushing and flossing. ${ }^{7}$ Demineralization generally follows fixed orthodontic treatment, if oral hygiene is not maintained. ${ }^{8}$ The reason for this are the bacteria's acidic residues in the plaque and it has negative impact on aesthetics. ${ }^{9}$ This study aims to assess the oral health awareness amongst orthodontic patients of Terna Dental College and to provide insight into oral hygiene awareness program.

\section{Materials and Methods}

The study sample consisted of 500 patients, $15-35$ years of age attending orthodontic OPD at Terna Dental College and Hospital, Navi Mumbai. A pilot study was initially conducted with 10 patients. They were asked to answer many questions relating to oral hygiene awareness. Furthermore, irrelevant questions were omitted. Worthy questions were used in main study. A self-made questionnaire was prepared consisting of questions which aided to assess oral hygiene awareness. Out of the 15 questions, 5 are related to knowledge, 5 are related to attitude and 5 are related to practice. After approval from concerned authorities, the preformed questionnaire was handed over to the subjects. They were explained the purpose of the study. After collection, the data was analysed using percentage.

Questionnaire: The present survey was conducted by simple random sampling method among 500 orthodontic patients from the Department of Orthodontics and Dentofacial Orthopaedics, Terna Dental College, Navi Mumbai, Maharashtra, India. Male and female patients were asked closed-ended questions. To assess awareness towards practices of oral hygiene in patients undergoing fixed Orthodontic treatment. Questions were given with answer choices that were easily understandable and brief. All the doubts that the participants were clarified. All answers were kept confidential. Patients who completed their minimum of 3 months of orthodontic treatment were included. The study was conducted from October 2017 to October 2018, which consisted of 15 questions assessing the awareness of oral health and the attitude of orthodontic correction-seeking individuals

Ethical Clearance: Ethical clearance was obtained from the institutional review board of Terna Public Charitable Trust Dental Hospital (TPCT) Ethical Committee. All answers were collected and recorded. All recorded data were analysed using percentage.

\section{Results}

In this survey, $65 \%$ were male and $35 \%$ were female. $100 \%$ of total sample used tooth brush as a method to clean 
their teeth (Fig. 1). 36\% of them were brushing once a day, $52 \%$ brushed their teeth twice a day, $12 \%$ were brushing thrice a day. $52.6 \%, 40 \%, 7.2 \%$ used soft, medium, hard material toothbrush respectively. $0.2 \%$ did not answer the question. (Fig. 2). 62\% of the patients used inter-dental aid to clean the proximal areas in which $16 \%$ used inter-dental brush, only $22 \%$ showed evidence of using dental floss, $16 \%$ used toothpick which should not be used, $2 \%$ used water- pick which is often recommended, $6 \%$ used combination of floss, inter-proximal brush and water pick which is the best approach (Fig. 3). Only 16\% used mouth wash and $74 \%$ rinsed with water post meal, 10\% didn't rinsed (Fig. 4). $48 \%$ were ok with their appearance with braces, $48 \%$ felt that they looked ugly, 4\% didn't not answer question regarding their appearance (Fig. 5).

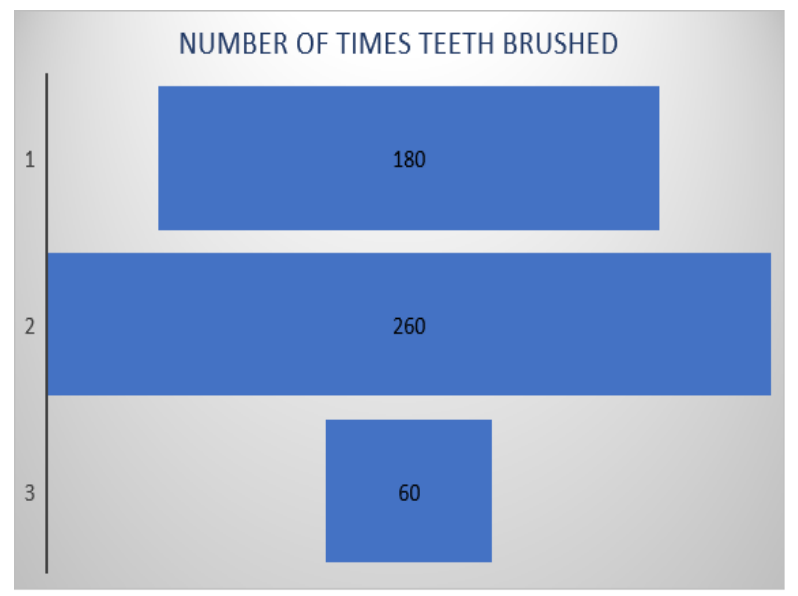

Fig. 1: Number of times sample's brushed their teeth

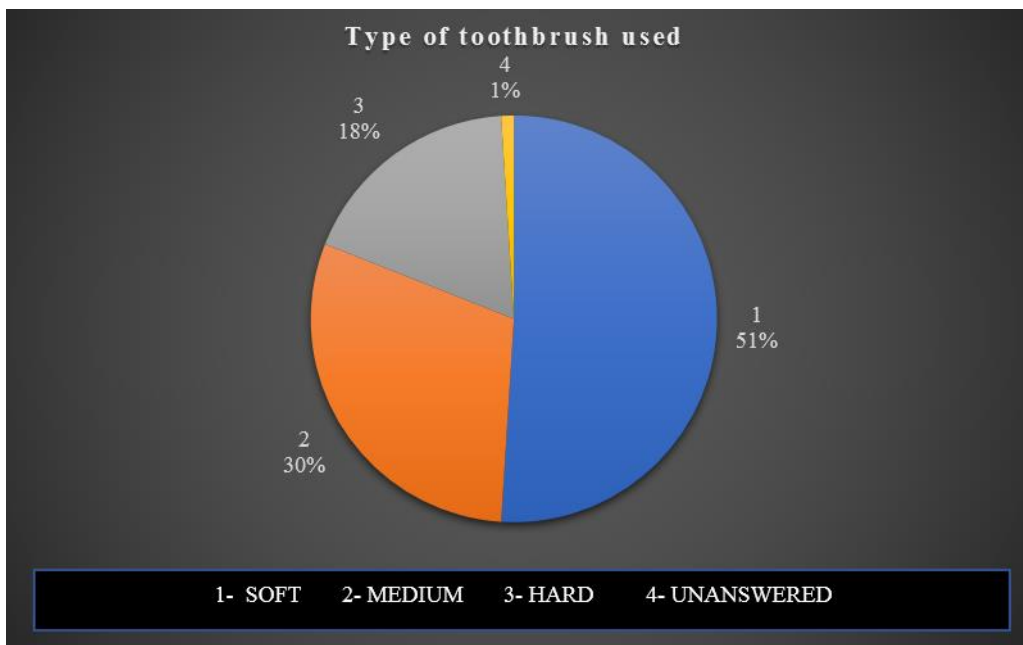

Fig. 2: Type of toothbrush used 


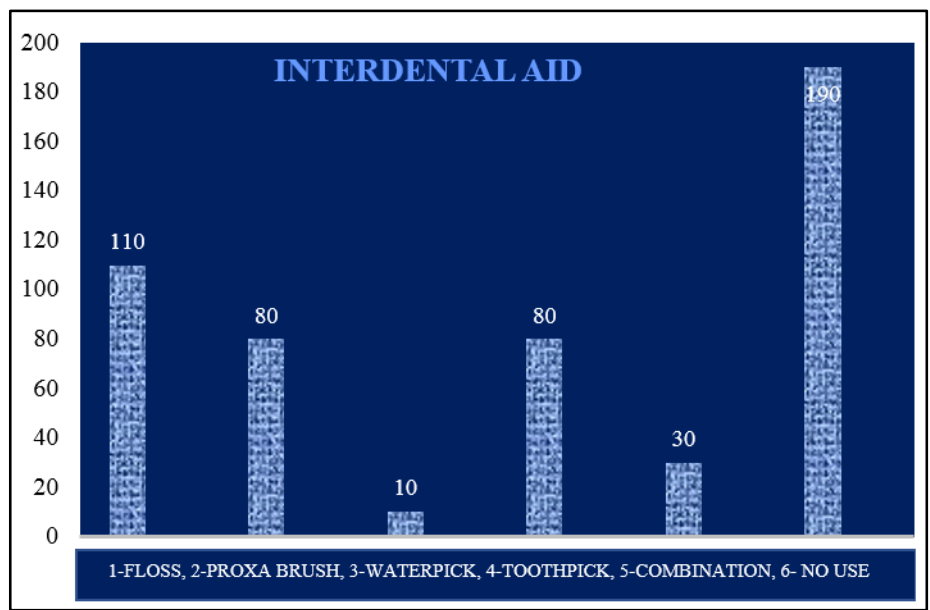

\section{Fig. 3: Type of inter-dental AID}

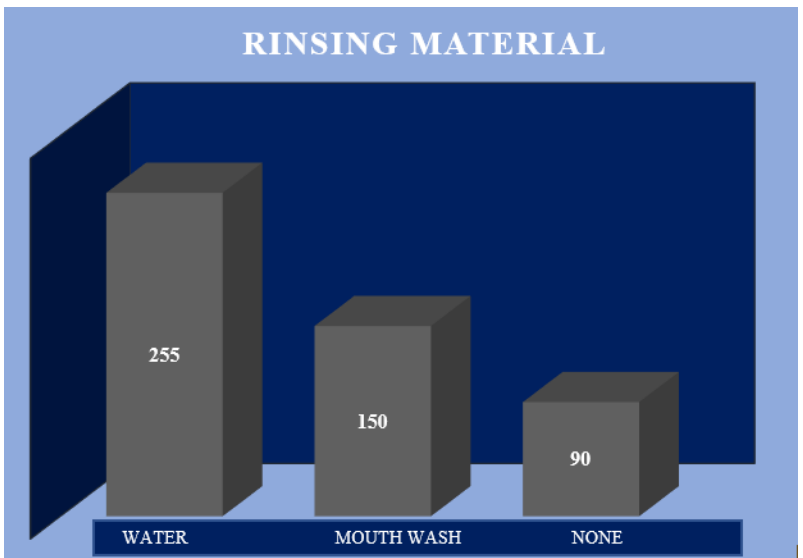

Fig. 4: Rinsing material used

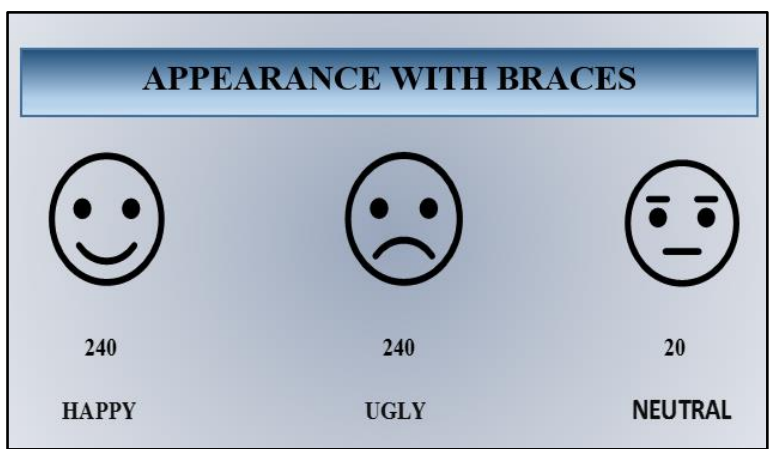

Fig. 5: Appearance WTH braces

\section{Questionnaire}

Questionnaire (Oral Hygiene in Fixed Orthodontic Patients)

Name - Age -

Gender - $\quad$ Occupation -

Address -

Socio-economic status - Upper class/Middle class/Lower Class

1. Do you brush your teeth?

Yes/No

2. If yes, then how many times?

$1 / 2 / 3$

3. Which material do you use to brush your teeth?
Tooth Paste/Neem Powder/Salt

4. What tool do you use to brush your teeth?

Tooth Brush/Finger/Else

5. If brush, then what type of brush?

Soft/Medium/Hard

6. What direction do you brush in?

Straight/ Horizontal/Vertical

7. Do you change your brush often?

Yes/No

8. If yes, then in how much time?

After 3 months/6 months/1 year

9. Do you use any tool to clean between your teeth? Yes/No

10. Do you use any of the following?

Floss/Toothpick/Inter-dental/Brush/Water pick

11. Do you rinse after eating food?

Yes/No

12. If yes, then with what?

Water/Mouth Wash

13. Have you ever had malodour?

Yes/No

14. Have you ever brushed very forcefully? Yes/No

15. Do you think your teeth look ugly with braces? Yes/No

\section{Discussion}

Oral hygiene is highly neglected in developing country like India. Preventive oral health knowledge and its implementation are the important ways of keeping our oral cavity healthy. Hence, in this study attempts were made to evaluate preventive oral health knowledge and its implementation by patients undergoing orthodontic treatment in Terna Dental College, Navi - Mumbai, Maharashtra, India. 100\% patients in this study used toothbrush for brushing, it was better than study in Department of Orthodontics and Dentofacial Orthopaedics, Rural Dental College, Loni, Maharashtra where 91.33\% used toothbrush. ${ }^{4}$ In a study for assessment of Oral Hygiene awareness in Geriatric patients, $70 \%$ uses toothpaste, $10 \%$ indicated use of neem stick and $20 \%$ used tooth powder 
with finger. ${ }^{10}$ The percentage of subjects brushing their teeth twice daily is $44 \%$ which is quite good as compared with $23 \%$ of the geriatric patients at Vyas Dental College and Hospital, Jodhpur but very less as compared to $75 \%$ of the elderly. ${ }^{11}$ This suggests that geriatric patient's frequency of brushing teeth is greater. Reason may be due to more amount of time for oneself in retired life. In this study, 22\% used floss as interdental aid which is poor finding as compared to Hamilton and were about $44 \%$ of the subjects studying in north eastern Ontario used dental floss. ${ }^{14}$ In contract, subject at Rural dental college only $6 \%$ subjects used floss. ${ }^{4}$ Reason for this may be the significant oral hygiene awareness programs that were carried out in Canada. This emphasizes the urgent need for educating and motivating the public to use this efficient method for oral health care in rural region. Furthermore, $90 \%$ subjects used to rinse after meals. It is much better than $39 \%$ of the sample population rinsing their mouth after eating food in rural dental college. Out of $90 \%, 16 \%$ subjects used mouth wash and $74 \%$ patients used water. They used it to treat malodour. Furthermore, $48 \%$ reported halitosis.

This study is in contrast with that of an epidemiologic survey of the general population of Japan where $24 \%$ of the individuals examined had complained about halitosis. ${ }^{14}$ Cost of mouth wash can be the reason for its less use. Regarding other oral hygiene practices, in a study by Berlin-Broner $e t$ al only $31.5 \%$ used mouthwash as an oral hygiene aid while in this study, only $31.33 \%$ of the individuals used mouthwash in their oral hygiene practice and $22.66 \%$ of the individuals used interdental toothbrush. Participants who received a post-treatment communication reported higher level of oral hygiene compliance than participants in the control group. $46 \%$ of subjects experienced malodour and $54 \%$ are aware that their teeth look dirty. ${ }^{10}$ The need for educating and motivating orthodontic patients on oral hygiene is elicited by the end of the study.

\section{Conclusion}

It is the need of an hour to establish and demonstrate a connection between good oral hygiene and its relation directly to overall health. We, as dentists, will have to keep reinforcing the importance brushing and Flossing along with the importance of regular check-ups. "Prevention is always better than cure". Adding mouthwash like Listerine as an adjunct to oral hygiene regimen may have positive effect on orthodontic patients in maintaining hygienic oral environment, thus reducing the likelihood for development of white spot lesions and gingival inflammation. To create oral hygiene awareness, various public health awareness programs through mediums such as camps, media, news and awareness events in public areas like railway and bus stations are encouraged. Such innovative methods of reaching the public not only ensure a healthy individual but a healthy over all society.
2. Guo L, Feng Y, Guo HG, Liu BW, Zhang Y Consequences of orthodontic treatment in malocclusion patients: clinical and microbial effects in adults and children. BMC Oral Health $16: 112$

3. Gilbert AD, Nuttal NM. Self-reporting of periodontal health status. Br Dent J 1999;186:241-244

4. Mayuresh J, Nandlal G, Survey on oral hygiene protocols among orthodontic correction-seeking individuals. Journal of Education and Ethics in Dentistry Wolters Kluwer Medknow

5. Eser T; Zachary A.; Steven J.; Chad E.; Kelly T. Effectiveness of an Essential Oil Mouth rinse in Improving Oral Health in Orthodontic Patients. Angle Orthodontist, Vol 78, No 2, 2008.

6. Dilip CL. Health status, treatment requirements, knowledge and attitude towards oral health of police recruits in Karnataka. J Indian Assoc Public Health Dent 2005;5:20-34

7. Hamilton ME, Coulby WM. Oral health knowledge and habits of senior elementary school students. J Public Health Dent 1991;51:212-218

8. Kamna S., Tripti T., Rohit K. and Kiran S. Risk factors and management of white spot lesions in Orthodontics. J Orthod Sci 2013;2(2).

9. Abhishek S., Aanchal S., Rohit K., Sahil G., Deepak P., Mayank G. Assessment of Oral Hygiene awareness in Geriatric patients attending OPD at ESIC Dental College, Rohini, New Delhi. J Oral Health Craniofacial Sci 2017;2:104-109.

10. Jain N, Mitra D, Ashok KP, Dundappa J, Soni S, et al. Oral hygiene-awareness and practice among patients attending OPD at Vyas Dental College and Hospital, Jodhpur. J Indian Soc Periodontol 2012;16:524-528.

11. Berlin-Broner Y, Levin L, Ashkenazi M. Awareness of orthodontists regarding oral hygiene performance during active orthodontic treatment. Eur J Paediatr Dent 2012;13:187-91.

12. Miyazaki H, Sakao S., Katoh Y., Takehara T. Oral malodour in the general population of Japan. Bad Breath: Research Perspectives. Tel Aviv Univ 1995;119-136.

13. Kallio P., Nordblad A., Croucher R., Ainamo J. Self-reported gingivitis and bleeding gums among adolescents in Helsinki. Community Dent Oral Epidemiol 1994;22:277-282.

14. Al-Shammari KF, Al-Ansari JM, Al-Khabbaz AK, Dashti A, Honkala EJ. Self-reported oral hygiene habits and oral health problems of Kuwaiti adults. Med Principle Pract 2007

15. Freeman R, Maizels J, Wyllie M, Sheiham A. The relationship between health-related knowledge, attitudes and dental health behaviors in 14-16-year-old adolescents. Community Dent Health 1993.

How to cite this article: Shah $\mathrm{K}$, Shenava $\mathrm{S}$, Kulshrestha R, Hawaldar C. Evaluation of oral hygiene and perception of patients undergoing orthodontic treatment attending OPD at Terna Dental College, Mumbai, Maharashtra. Int Dent J Student's Res. 2018;6(4): 81-84.

\section{References}

1. Axelson P. Current role of pharmaceuticals in prevention of caries and periodontal disease. Int Dent J 43:473-482. 